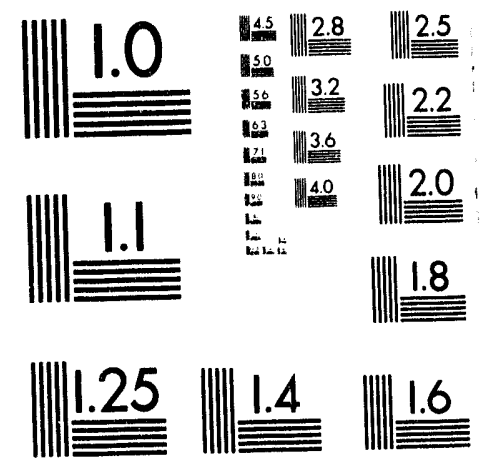



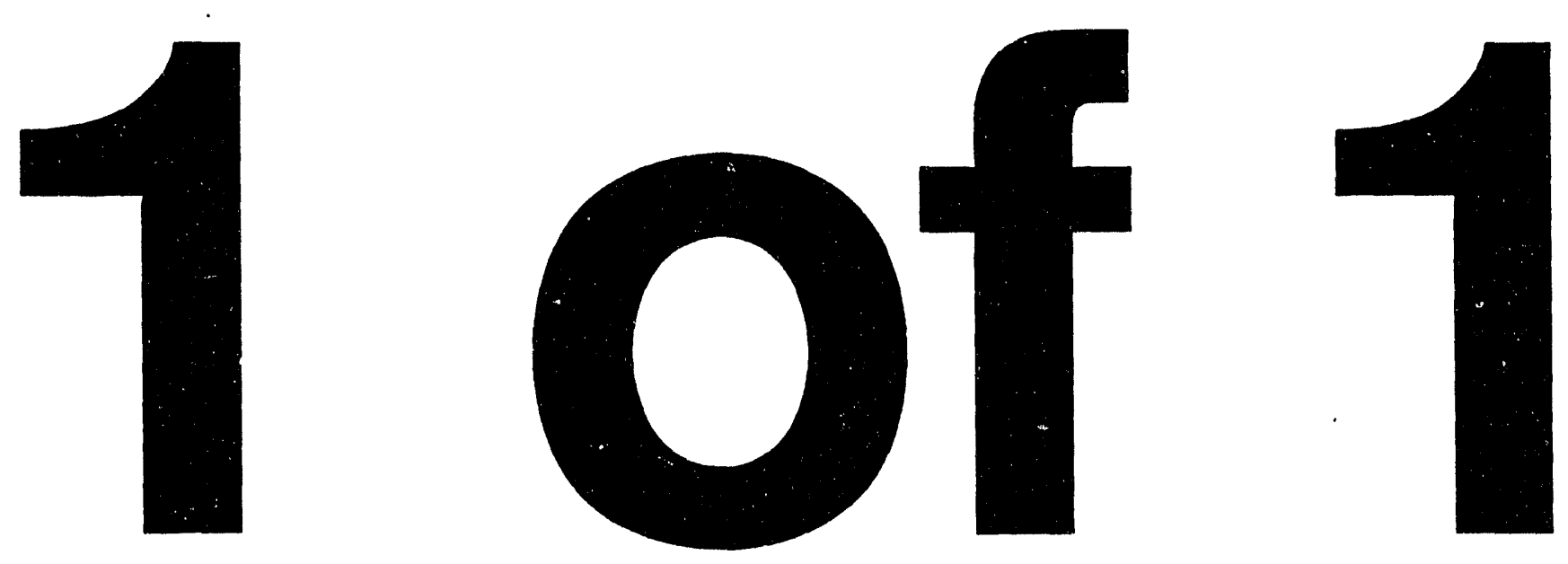


\title{
Supersymmetric Renormalisation Group Fixed Points and Third Generation Fermion Mass Predictions
}

\author{
C D Froggatt ${ }^{1}$ and R G Moorhouse1 \\ Department of Physics and Astronomy, \\ University of Glasgow, Glasgow C12 8QQ, UK \\ and \\ IG Knowles 2 \\ High Energy Physics Division \\ Argonne National Laboratory, 9700 South Cass Avenue \\ Argonne, Il 60439, USA
}

\begin{abstract}
We present a supersymmetric renormalisation group fixed point determination of the third generation fermion masses, in which the large mass ratio between the top and bottom quarks is attributed to a hierarchy in the vacuum expectation values of the two Higgs doublets. Above a supersymmetry breaking scale, $M_{s}$, we use the minimal supersymmetric standard model with a transition at $\mathrm{M}_{\mathrm{s}}$ to the standard model with only one Higgs doublet effective. The mass predictions result from renormalisation group evolution of large Yukawa couplings at $\mathrm{M}_{\mathrm{x}} \sim 10^{16} \mathrm{GeV}$. Averaging over a wide range of these couplings, not subject to any symmetry requirements, gives

$\mathrm{m}_{\mathrm{t}}=184.3 \pm 6.8 \mathrm{GeV}, \mathrm{m}_{\mathrm{b}}=4.07 \pm 0.33 \mathrm{GeV}, \mathrm{m}_{\tau}=1.78 \pm 0.33 \mathrm{GeV}$ and a light Higgs mass $m_{h} 0=121.8 \pm 4.3 \mathrm{GeV}$ for $M_{s}=1 \mathrm{TeV}$ and $\alpha_{s}\left(M_{z}\right)=0.125$.
\end{abstract}

\section{DISCLAIMER}

\begin{abstract}
This report was prepared as an account of work sponsored by an agency of the United States Government. Neither the United States Government nor any agency thereof, nor any of their employees, makes any warranty, express or implied, or assumes any legal liability or responsibility for the accuracy, completeness, or usefulness of any information, apparatus, product, or process disclosed, or represents that its use would not infringe privately owned rights. Reference herein to any specific commercial product, process, or service by trade name, trademark, manufacturer, or otherwise does not necessarily constitute or imply its endorsement, recommendation, or favoring by the United States Government or any agency thereof. The views and opinions of authors expressed herein do not necessarily state or reflect those of the United States Government or any agency thereof.
\end{abstract}

1 Research partially funded by the UK Science and Engineering Research Council

2 Work supported by the US Department of Energy, Division of High Energy Physics, Contract W-31-109-ENG-38 
Limits on the top quark mass from experiment and radiative corrections $[1,2]$ have revived interest in the idea that its value corresponds to an infra-red fixed point $[3,4,5,6]$ of the renormalization group equations. Assuming the desert hypothesis of no new interactions beyond the standard model up to very high energies, $M_{X} \sim 10^{15}-10^{19} \mathrm{GeV}$, the effective fixed point $[6,7]$ for three generations predicts the top quark mass to be $m_{t} \approx 225 \mathrm{GeV}$, together with the mass of the Higgs $m_{h} \approx 250 \mathrm{GeV}$. In addition to the desert hypothesis it is necessary to assume that the top quark Yukawa coupling constant is large (greater than unity) at the high energy scale $\mu \approx M_{x}$ in order to be within the basin of attraction of the infra red fixed point. However the value of the Higgs self-coupling constant $\lambda$ at $\mu=M_{x}$ does not have to be constrained; it must simply be positive semi-definite. The large coupling of the Higgs doublet to the top quark is sometimes interpreted $[7,8]$ to mean that the Higgs particle is a $\mathfrak{t}$ bound state with a compositeness scale $\mu \sim \mathrm{M}_{\mathbf{x}}$. But the predicted high value of the top quark mass $\mathrm{m}_{\mathrm{t}} \approx 225 \mathrm{GeV}$ appears to be inconsistent with the upper limit $\mathrm{m}_{\mathrm{t}}<200 \mathrm{GeV}$ derived from precision LEP measurements and standard model radiative corrections [2].

Renormalisation group fixed points have also been considered for two $\mathrm{Higgs}$ doublet models $[6,9,10]$. With two Higgs doublets it is possible for both the top and bottom quark masses to show fixed point behaviour, provided that the ratio of the scalar field vacuum expectation values is fine tuned so that $\mathrm{v}_{2} / \mathrm{v}_{1} \approx \mathrm{m}_{\mathrm{b}} / \mathrm{m}_{\mathrm{t}}$. In this way we trade the mystery of the bottom to top quark mass ratio for that of a hierarchy of vacuum expectation values (VEVs). This hierarchy of vacuum values would also help account for the fact that the charm quark is much heavier than the strange quark, but not for the light quark mass ratio $m_{d} / m_{u}$. However the motivation for the use of a hierarchy of VEVs in this paper is that it allows a fixed point model prediction for the b quark to tau lepton mass ratio as well as for the top quark mass.

In some previous papers $[10,11,12]$ the authors have investigated such a theory with a general Higgs scalar potential without supersymmetry or any special grand unified theory structure at high energies. One positive feature of these investigations is that for a large range of randomly chosen Yukawa couplings (greater than 1 ) and scalar couplings at high energies $\left(\mathbf{M}_{\mathbf{X}} \approx 10^{15}\right.$ $\mathrm{GeV}$ ), the renormalisation group equations give an $m_{b} / m_{\tau}$ ratio of $2.6 \pm 0.8$. However, with the scalar potential respecting the usual custodial discrete symmetry required to ensure tree level natural flavour conservation and $v_{2} \ll v_{1}$, a low mass scalar $\eta_{0}$ with $5 \mathrm{GeV} \geq \mathrm{m}_{\eta} 03.5$ $\mathrm{GeV}$ results [10] in disagreement with data on radiative upsilon decay [14]. To cure this a term in the scalar potential which softly breaks the discrete symmetry is introduced $[10,11]$ and since it may break CP invariance it has its own intrinsic interest $[15,16,17]$. Nonetheless, for $v_{2} \ll v_{1}$, the predicted top quark mass, $m_{t} \approx 220 \mathrm{GeV}$, remains too high, generating large radiative corrections which are not compensated by the contributions from the spectrum of Higgs scalar particles [11]. For $v_{2} \approx v_{1}$ the fixed point top quark mass is of order $160 \mathrm{GeV}$ and the radiative corrections are within the range indicated by the present data, but there is no fixed point prediction for $m_{b} / m_{\tau}$.

In this letter we consider the implications of importing supersymmetry into the above scenario. There are several motivations for considering the minimal supersymmetric extension of the standard model: 1) Supersymmetry can cure the technical gauge hierarchy problem; the 
smallness of the electroweak scale relative to the high energy cut-off scale associated with new physics, ie $M_{w} / M_{x} \ll 1$, is protected from large radiative corrections provided the superpartners of the standard model particles exist in or below the $\mathrm{TeV}$ mass range. However the minimal supersymmetric standard model [18] joes not explain why $M_{w} / M_{x}$ is so small to start with and it is necessary to assume the supersymmetry breaking scale $M_{s}$ is of order 1 $\mathrm{TeV}$. 2) Unification of the standard model gauge coupling constants can be obtained by including all the superpartners contained in the minimal supersymmetric model [19], consistent with $\mathrm{M}_{\mathrm{x}} \approx 10^{16} \mathrm{GeV}$ and $\mathrm{M}_{\mathrm{s}} \sim 1 \mathrm{TeV}$. It should be emphasised however that the unification requirement does not fix $M_{s}$ with any precision; $M_{s}$ has an uncertainty of many orders of magnitude [20]. 3) Minimal supersymmetry requires two complex Higgs doublets $\Phi_{1}$ and $\Phi_{2}$ which couple to the top quark and to the bottom quark and tau lepton respectively; this is precisely the Higgs structure assumed above. 4) The fixed point value of the top quark Yukawa coupling constant in the minimal supersymmetric model is smaller than in the standard model $[21,22]$. This last point is pragmatically the main reason for introducing supersymmetry into our model.

We now reconsider our fixed point model with $v_{2} \ll v_{1}$ in the context of the minimal supersymmetric mudel broken at $\mathrm{M}_{s} \sim 1 \mathrm{TeV}$. The soft symmetry breaking terms in the two Higgs potential generate approximately equal masses of order $M_{s}$ for the 'extra' scalar particles and below this scale there is an effective one doublet Higgs system $[11,12,23]$ with $m_{h} 0<$ $M_{s}$. Barbieri et al [24] have investigated the dependence of the light Higgs mass $m_{h} 0$ on the top quark mass $m_{t}$, using the renormalisation group equations, assuming that all the extra particles introduced by supersymmetry have effectively degenerate masses of $M_{s}=1 \mathrm{TeV}$. Assuming the desert hypothesis between $M_{s} \sim 1 \mathrm{TeV}$ and $\mathrm{M}_{\mathrm{x}} \approx 10^{16} \mathrm{GeV}$ we investigate infra red fixed point predictions for the third generation fermion masses.

The renormalisation group equations for the minimal supersymmetric model are used for $\mathrm{M}_{\mathrm{s}}<\mu<\mathrm{M}_{\mathrm{x}}$ and the standard model renormalisation group equations are used for $\mu<\mathrm{M}_{\mathrm{s}}$. All the new supersymmetric particles are taken as effectively degenerate in mass at the supersymmetry breaking scale $\mathrm{M}_{\boldsymbol{s}}$. Wo do not make any assumptions about the nature of the unification symmetry or the Higgs structure at $M_{x} \approx 10^{16} \mathrm{GeV}$. In particular, unlike in other related work $[22,25]$, we do not assume a symmetry relationship between the running fermion masses, such as $m_{b}=m_{\tau}$, at $\mu=M_{x}$. The Yukawa couplings of the third generation fermions are taken to be greater than unity but otherwise random at $\mu=M_{x}$ within a region which we specify more precisely below. Using these random starting values at $\mu=M_{x}$ robust predictions for $m_{t}, m_{h}$ and the ratio $m_{b} / m_{\tau}$ are obtained. Again it is possible to interpret the large values of the Yukawa coupling constants at $\mu=M_{x}$ in terms of composite Higgs fields and Carena et al [26] have considered such a supersymmetric top quark condensate model, in which just the top quark mass takes a fixed point value depending on the ratio $v_{2} / v_{1}$ which is taken to be greater than $m_{b} / m_{\imath}$.

In the minimal supersymmetric model the up quarks couple to a Higgs doublet $\Phi_{1}$ while the 
down quarks and leptons couple to another Higgs doublet $\Phi_{2}$. We adopt the notation $g_{i}$ $(i=1,2,3)$ and $g_{f}(f=t, b, \tau)$ for the gauge and Yukawa couplings respectively, with $D \equiv 16$ $\pi^{2} \mathrm{~d} / \mathrm{d}(\ln \mu)$ where $\mu$ is the renormalization scale.

Then the renormalization group equations for $\mu>M_{s}$ are $[21,27]$

$$
\begin{aligned}
& D g_{i}=-b_{i} g_{i}^{3} \text { and } D g_{f}=g_{f} B_{f} \\
& b_{1}=-11, b_{2}=-1, b_{3}=3 \\
& B_{t}=\left(6 g_{t}{ }^{2}+g_{b}{ }^{2}-16 g_{3}{ }^{2} / 3-3 g_{2}^{2}-13 g_{1}{ }^{2} / 9\right) \\
& B_{b}=\left(g_{t}{ }^{2}+6 g_{b}{ }^{2}+g_{\tau}{ }^{2}-16 g_{3}^{2} / 3-3 g_{2}{ }^{2}-7 g_{1}{ }^{2} / 9\right) \\
& B_{\tau}=\left(3 g_{b}{ }^{2}+4 g_{\tau}{ }^{2}-3 g_{2}{ }^{2}-3 g_{1}{ }^{2}\right)
\end{aligned}
$$

For $\mu>M_{s}$ the scalar potential is given by the minimal supersymmetric model [18] in which we take all the superpartners to be degenerate in mass and equal to $M_{s}$. At $\mu=M_{s}$ all the extra particles drop out of the theory and we join [24] to the standard model with one Higgs scalar, $\phi$, having potential

$$
V=\frac{1}{4} \lambda\left(\phi^{2}-v^{2}\right)^{2}
$$

where $v^{2}=v_{1}^{2}+v_{2}^{2}=(246 \mathrm{GeV})^{2}$ and

$$
\lambda\left(M_{s}\right)=\left(\frac{v_{1}^{2}-v_{2}^{2}}{v^{2}}\right)^{2}\left(g_{1}^{2}\left(M_{s}\right)+g_{2}^{2}\left(M_{s}\right)\right) / 8
$$

We require also that the fermion masses (above $M_{s}$ given by $g_{t} v_{1} / \sqrt{2}, g_{b} v_{2} / \sqrt{2}$ and $g_{\tau} v_{2} / \sqrt{2}$ respectively) be continuous so that the Yukawa couplings change as

$$
g_{t} \rightarrow g_{t} v_{1} / v, g_{b} \rightarrow g_{b} v_{2} / v, g_{\tau} \rightarrow g_{\tau} v_{2} / v
$$

We then continue for $\mu<M_{s}$ with the renormalization group equations given by (1) with

$$
b_{1}=-41 / 6, \quad b_{2}=19 / 6, \quad b_{3}=7
$$

and by 


$$
\begin{aligned}
& \mathrm{B}_{\mathrm{t}}=\left(9 \mathrm{~g}_{\mathrm{t}}^{2} / 2+3 \mathrm{~g}_{\mathrm{b}}{ }^{2 / 2}+\mathrm{g}_{\tau}{ }^{2}-8 \mathrm{~g}_{3}{ }^{2}-9 \mathrm{~g}_{2}{ }^{2 / 4-17} \mathrm{~g}_{1}{ }^{2 / 12}\right) \\
& \mathrm{B}_{\mathrm{b}}=\left(9 \mathrm{~g}_{\mathrm{b}}{ }^{2} / 2+3 \mathrm{~g}_{\mathrm{t}}{ }^{2} / 2+\mathrm{g}_{\tau}{ }^{2}-8 \mathrm{~g}_{3}{ }^{2}-9 \mathrm{~g}_{2}{ }^{2} / 4-5 \mathrm{~g}_{1}{ }^{2} / 12\right) \\
& \mathrm{B}_{\tau}=\left(3 \mathrm{~g}_{\mathrm{t}}{ }^{2}+3 \mathrm{~g}_{\mathrm{b}}{ }^{2}+5 \mathrm{~g}_{\tau}{ }^{2} / 2-9 \mathrm{~g}_{2}{ }^{2} / 4-15 \mathrm{~g}_{1}{ }^{2} / 4\right)
\end{aligned}
$$

together with

$$
\begin{aligned}
D \lambda= & 24 \lambda^{2}+\lambda\left(12 g_{b}{ }^{2}+12 g_{t}{ }^{2}+4 g_{\tau}{ }^{2}-9 g_{2}{ }^{2}-3 g_{1}{ }^{2}\right) \\
& +9 g_{2}{ }^{4} / 8+3 g_{2}{ }^{2} g_{1}{ }^{2} / 4+3 g_{1}{ }^{4} / 8-6 g_{b}{ }^{4}-6 g_{l}{ }^{4}-2 g_{\tau}{ }^{4}
\end{aligned}
$$

As $\mu$ comes down through the physical masses of the various particles, these drop out of the renormalization group equations [28]. The mass of the top quark is given by

$$
m_{t}=g_{t}\left(\mu=m_{t}\right) v / \sqrt{ } 2
$$

Below the scale of $m_{t}$ we set $g_{t}=0$ in equations (8b) (8c) and (9) and we take $b_{3}=7.667$. The mass of the Higgs particle $m_{h} 0$ is given by

$$
m_{h^{\circ}}^{2}=2 \lambda\left(\mu=m_{h^{0}}\right) v^{2}
$$

and below this scale the Higgs particle and its self-coupling constant $\lambda$ drop out of the equations.

Similarly below the scale of the weak gauge bosons we only run two gauge couplings, these being $\mathrm{g}_{3}$ and $\mathrm{e}$, the electromagnetic coupling constant which satisfies the matching condition

$$
e\left(M_{z}\right)=\frac{g_{1}\left(M_{z}\right) g_{2}\left(M_{z}\right)}{\left(g_{1}^{2}\left(M_{z}\right)+g_{2}^{2}\left(M_{z}\right)\right)^{1 / 2}}
$$

The renormalisation group equation for the electromagnetic coupling constant is

$$
\mathrm{De}=-\mathrm{b}_{\mathrm{e}} \mathrm{e}^{3}
$$

where

$$
b_{e}=-\frac{4}{3} \sum_{i} Q_{i}^{2}=-\frac{80}{9}
$$

for $m_{b}<\mu<M_{z}$. The QCD beta function remains unaltered in this region with $b_{3}=7.667$. The renormalisation group equations for the running masses $m_{b}$ and $m_{\tau}$ over the same energy 
scale are

$$
D m_{f}=m_{f} B_{f}
$$

where

$$
B_{b}=-\frac{2}{3} e^{2}-8 g_{3}^{2}, \quad B_{\tau}=-6 e^{2}
$$

The results are relatively insensitive to the precise values of $\mu$ at which the above scales are introduced; in the results given we choose to drop the top quark at $\mu=190 \mathrm{GeV}$ and drop the Higgs particle and change the gauge couplings at $\mu=\mathrm{M}_{\mathrm{z}}=91.2 \mathrm{GeV}$.

We run the masses of the bottom quark $m_{b}(\mu)$ and the tau lepton $m_{\tau}(\mu)$ down to the bottom quark mass given by

$$
m_{b}=m_{b}\left(\mu=m_{b}\right)
$$

This is not a prediction of the bottom quark mass (coming from the high energy values) because it depends on the ratio $v_{2} / v_{1}$ which occurs in the transition at the supersymmetry breaking scale given in eq (6). Similarly for the $\tau$ mass. However it is our hypothesis that $v_{2}$ is small: $v_{2}<10 \mathrm{GeV}, v_{2} / v_{1}<.04$ and within such a range the ratio $R=m_{b}\left(\mu=m_{b}\right) / m_{\tau}(\mu=$ $m_{b}$ ) is independent of the particular value of $v_{2}$. In other words given the experimental value of $m_{\tau}$, there is a prediction of $m_{b}\left(\mu=m_{b}\right)$.

In our calculations we employ values for the $U(1), S U(2)$ and $S U(3)$ gauge couplings as specified by experiment: [29] at the scale $M_{z}=91.184 \pm 0.007 \mathrm{GeV}$. Given [30] $\alpha_{\mathrm{EM}}^{-1}=127.9 \pm 0.2$ and [29] $\sin ^{2} \theta_{\text {eff }}=0.2324 \pm 0.0011$ we use equation (12) and the relation $\tan \theta=\mathrm{g}_{2} / \mathrm{g}_{1}$ to fix $\mathrm{g}_{1}=0.358$ and $\mathrm{g}_{2}=0.649$. Unfortunately the strong coupling is less well specified [31]: possible values range from $\alpha_{s}=0.105 \pm 0.004$ using the lattice [32] or $0.108 \pm 0.007$ using Upsilon decay [33] to $\alpha_{s}=0.135 \pm 0.011$ using the LEP line shapes [29] and beyond. Allowing for the uncertainty we consider $\alpha_{s}$ in the range $0.100-0.150$, equivalent to $g_{3}=1.121-1.373$. Note a weighted average of all experimental measurements gives [31] $\alpha_{\mathrm{s}}=0.118\left(\mathrm{~g}_{3}=1.218\right)$.

Since we are assuming the validity of perturbation theory we use a range of Yukawa couplings bounded above by $\sqrt{4 \pi^{2}}$ at the high scale $M_{x}$. Our results are based on an average and root mean square deviation over an integer valued starting grid with range:

$$
1 \leq \mathrm{g}_{\tau} \leq 6, \quad 1 \leq \mathrm{g}_{\mathrm{b}} \leq 6,1 \leq \mathrm{g}_{\mathrm{t}} \leq 6
$$


Defining $R=m_{b}(\mu) / m_{\tau}(\mu)$ with $\mu=4 \mathrm{GeV}$, the lower scale at which evolution is stopped, we find for $\alpha_{s}=0.125\left(g_{3}=1.253\right)$ independently of $v_{2} / v_{1}$.

$$
\mathrm{m}_{\mathrm{t}}=184.3 \pm 6.8 \mathrm{GeV}, \quad \mathrm{m}_{\mathrm{h}} \mathrm{o}=121.8 \pm 4.3 \mathrm{GeV}, \quad \mathrm{R}=2.42 \pm 0.74
$$

This value for the top quark mass, though somewhat high, is within two standard deviations of the result predicted from global analysis of experimental data $[2,11,29]$. Finally we tune $v_{2}$ so that the average value of $m_{\tau}$ is exactly equal to the experimental value [34] $1.7769 \pm 0.0007$ GeV, finding:

$$
v_{2}=3.86 \mathrm{GeV}, \quad m_{b}=4.07 \pm 0.33 \mathrm{GeV}, \quad m_{\tau}=1.78 \pm 0.33 \mathrm{GeV}
$$

Note that the error introduced by not running $g_{\tau}$ from $m_{b}$ to $m_{\tau}$ is significantly less than $1 \%$ and can be safely neglected.

Of course there is some uncertainty in specifying our predictions due to the possible use of alternative definitions. In order to assess the sensitivity to such ambiguities we investigated three methods of defining our results and also use three sets of initial starting values for the Yukawa couplings. This also serves to elucidate the role of the quasi-fixed point in determining our mass predictions. Our results are summarised in table 1 , for which $\alpha_{s}=$ $0.125\left(g_{3}=1.253\right)$ and $M_{s}=1 \mathrm{TeV}$.

At $\mu=M_{x}$ three options for the size of the uniform starting grid for the Yukawa couplings were considered: $(1-6)^{3}$ as in equation (18) above, $(3-6)^{3}$ and $(1-3)^{3}$. In each case three alternative prescriptions for adjusting $v_{2}$ and obtaining predictions and errors were then pursued. (A) $v_{2}$ was tuned so that the central value for $m_{\tau}$ after evolution equalled the experimental value, with errors specified as half the range spanned by the maximum and minimum values. (B) $v_{2}$ was tuned to ensure that the statistical average of $m_{\tau}$ is correct, with an error given by the standard deviation; this corresponds to the method employed above. (C) $v_{2}$ was tuned point-by-point so that the correct $m_{\tau}$ obtains for each starting point in the initial grid, the average and standard deviation of these individual runs are then quoted.

As expected, for each initial range, case (A) gives the largest errors and, except for $m_{b}$, there is little difference between the errors given by methods (B) and (C). Note however that for $\mathrm{M}_{\mathrm{h}} \mathrm{O}$ and $m_{t}$ the central value from $(A)$ is less than the average values from $(B)$ and $(C)$. This indicates that the majority of the corresponding final Yukawa couplings lie in the upper half of the total range spanned and is suggestive of the quasi-fixed point's presence. To confirm this observe that using the $(3-6)^{3}$ sub-range, whose points preferentially converge to the fixed point, gives higher mass predictions with significantly smaller errors. Further using the $(1-3)^{3}$ sub-range we find slightly lower mass predictions but with errors almost comparable in size with the full range $(1-6)^{3}$.

Next in fig. 1 we show the dependence of $m_{t}, m_{h} o$ and $m_{b}$ on the imprecise experimental input $\alpha_{s}\left(M_{2}\right)$. Method $B$ above is used to specify $v_{2} ; g_{1}, g_{2}$ and $M_{s}$ are fixed as $0.358,0.649$ and 
$1 \mathrm{TeV}$ respectively and the $\alpha_{\mathrm{s}}$ range is taken to be 0.10 to $0.15\left(\mathrm{~g}_{\mathrm{s}}=1.121-1.373\right)$. Both $\mathrm{m}_{\mathrm{t}}$ and $m_{h}$ o show only minor variation with $\alpha_{s}\left(M_{2}\right)$, which is approximately linear in $g_{3}$ over the range considered. This is not the situation for $m_{b}$ which shows a marked increase, from 3.42 to $4.87 \mathrm{GeV}$ with $\alpha_{s}$. For all three masses shown the errors remain essentially constant.

As previously noted the supersymmetry breaking scale $\mathrm{M}_{\mathrm{s}}$ is not well deiermined, even within gauge coupling unification scenarios. To investigate the dependence of our predictions on this ambiguity we consider varying $M_{s}$ between $200 \mathrm{GeV}$ and $5 \mathrm{TeV}$. The results for $m_{t}, m_{h} 0$ and $m_{b}$ are shown in fig. 2, where again method $B$ has been used to determine $v_{2}$ and $\alpha_{s}=0.125$ $\left(g_{s}=1.253\right)$. The fermion masses $m_{t}$ and $m_{b}$ show only a modest dependence, which is well described as linear in $\log \left(M_{s}\right)$. However the Higgs mass $m_{h} 0$ is sensitive to $M_{s}$, increasing from 91.7 to $141.9 \mathrm{GeV}$ as $\mathrm{M}_{\mathrm{s}}$ rises from 200 to $5000 \mathrm{GeV}$; a corresponding rise in the error estimate is also noted. The $m_{h} 0$ dependence is not logarithmic.

To summarise, our results have been shown to be stable to minor modifications in the details of the method employed. We have also demonstrated that using larger starting values for the initial starting grid at $\mathrm{M}_{\mathbf{x}}$ emphasises the role of the quasi-fixed point and significantly reduces our error estimates. The $M_{s}$ dependence of $m_{t}, m_{h} o$ and $m_{b}$ has been investigated: larger predicted values obtaining for higher supersymmetry breaking scales, but with this effect only being significant for the Higgs mass. Also considered was the effect of varying $\alpha_{s}\left(M_{z}\right)$. A small increase with $\alpha_{s}$ is seen in $m_{t}$ and through equation (9) this leads to a lesser dependence in $m_{h} 0$. However as suggested by equations (15) and (16), $m_{b}(\mu)$ is rather sensitive to $\alpha_{s}(\mu)$ in particular because at the relevant lower scale, $\mu=4 \mathrm{GeV}, \alpha_{s}$ has started to grow significantly.

The results presented here are consistent with those given in a preliminary report submitted to the 1992 ICHEP, Dallas meeting [35].

The experimental bottom quark mass is not well determined. Values have been extracted from QCD sum rules [36] and analyses of charmonium and B meson spectra [37]. Calculations using $\mathrm{QCD}$ sum rules give $\mathrm{m}_{\mathrm{b}}\left(\mathrm{m}_{\mathrm{b}}\right)=4.25 \pm 0.10 \mathrm{GeV}$, but the quoted error probably underestimates the intrinsic uncertainty in this approach. It is difficult to establish the connection between $m_{b}\left(m_{b}\right)$ and the constituent quark mass derived from analyses of charmonium and B-meson spectra; but naive estimates of confinement effects have been used to give values of $m_{b}\left(m_{b}\right)$ in the range $4.7 \pm 0.3 \mathrm{GeV}$. Our results are consistent with the lower value $m_{b}\left(m_{b}\right) \approx 4.25 \mathrm{GeV}$ obtained from $Q C D$ sum rules.

Other predictions for the $b$ quark mass in the literature are usually based on the presumed symmetry relation $g_{b}\left(M_{x}\right)=g_{\tau}\left(M_{x}\right)$. This symmetry relationship is motivated by the minimal $\mathrm{SU}(5)$ grand unified model with the Higgs particle in a 5 dimensional representation, giving $\mathrm{m}_{\mathrm{b}}$ $\left(m_{b}\right) / m_{\tau}\left(m_{b}\right) \approx 3$ in approximate agreement [38] with the upper limit $m_{b}\left(m_{b}\right)=5 \mathrm{GeV}$ of the 'experimental' range mentioned above. In fact running the symmetry relation from $\mu=M_{x}$ to $\mu=m_{b}\left(m_{b}\right)$ using the standard model renormalisation group equations gives a value closer to 
$5.5 \mathrm{GeV}$; this value is further increased[39] if (i) a top quark mass greater than $100 \mathrm{GeV}$ is used and/or (ii) extra non-supersymmetric matter fields are introduced in the desert to ensure unification of the gauge coupling constants. In a two Higgs model, however, the effect of a large Yukawa coupling constant for the top quark is to reduce the predicted value of $m_{b}\left(m_{b}\right)$. A b quark mass $m_{b}\left(m_{b}\right)$ of $5 \mathrm{GeV}$ can thereby be obtained with a non-supersymmetric $S U(5)$ unification, provided that the top quark Yukawa coupling constant is close to its infra-red fixed point [39]. Similarly, within supersymmetric grand unification, the symmetry relation $g_{b}\left(M_{x}\right)$ $=g_{\tau}\left(M_{x}\right)$ leads to $m_{b}\left(m_{b}\right) \approx 4.25 \mathrm{GeV}$ for $M_{s}=1 \mathrm{TeV}$ and $g_{t}\left(m_{t}\right)$ close to its infra red fixed point $[22,25]$.

We emphasise again that our model does not use any grand unification symmetry relation, such as $g_{b}\left(M_{x}\right)=g_{\tau}\left(M_{x}\right)$. Our assumptions are: 1) The minimal supersymmetric standard model is valid at energies greater than $M_{s} \approx 1 \mathrm{TeV}$. 2) There are no new interactions beyond the minimal supersymmetric model in the desert between $M_{s} \approx 1 \mathrm{TeV}$ and $\mathrm{M}_{x} \approx 10^{16} \mathrm{GeV} .3$ ) The Yukawa coupling constants for the $t$ quark, $b$ quark and tau lepton are greater than unity but otherwise unrelated at the scale $\mu=M_{\mathbf{x}}$. This last assumption implies a hierarchy of vacuum values $v_{2} \ll v_{1}$ and infra red effective fixed point behaviour $[6,10]$ for both the top and bottom quarks. In practice the trajectories for $g_{\tau}(\mu)$ also cluster around a non-zero value in the infra red.

In conclusion the supersymmetric renormalisation group fixed point third generation quarklepton spectrum is phenomenologically attractive. Averaging over a wide range of large Yukawa couplings at $M_{x} \approx 10^{16} \mathrm{GeV}$ and choosing $v_{2}$ so that the average value of $m_{\tau}$ agrees with experiment, we find for $\alpha_{s}\left(M_{z}\right)=0.125$ and $M_{s}=1 \mathrm{TeV}: m_{t}=184.3 \pm 6.8 \mathrm{GeV}, m_{b}=$ $4.07 \pm 0.33 \mathrm{GeV}, \mathrm{m}_{\tau}=1.78 \pm 0.33 \mathrm{GeV}$ and $\mathrm{m}_{\mathrm{h}} \mathrm{o}=121.8 \pm 4.3 \mathrm{GeV}$. 


\section{References}

1. CDF Collaboration: F Abe et al., Phys. Rev.Lett. 68 (1992) 447

2. G Altarelli, R Barbieri and S Jadach, Nucl. Phys. B369 (1992) 3;

$\mathrm{J}$ Carter, Proc. of the LEP-HEP 91 Conference, Eds. S Hegarty, K Potter and

E Quercigh, p 1 (World Scientific, 1992)

J Ellis, ibid, $p 27$

3. C D Froggatt and H B Nielsen, Nucl. Phys. B147 (1979) 277

4. N Cabibbo, L Maiani, G Parisi and R Petronzio, Nucl. Phys. B158 (1979) 295

5. B Pendleton and G G Ross, Phys. Lett. B98 (1981) 291

6. C T Hill, Phys. Rev. D24 (1981) 691; C T Hill, C N Leung and S Rao, Nucl. Phys. B262 (1985) 517

7. W A Bardeen, C T Hill and M Lindner, Phys. Rev. D41 (1990) 1647

8. A Hasenfratz, P Hasenfratz, J Kuti and Y Shen, Nucl. Phys. B365, (1991) 79

9. M Luty, Phys. Rev. D41, (1990) 2893

10. C D Froggatt, I G Knowles and R G Moorhouse, Phys. Lett. B249 (1990) 273

11. C D Froggatt, I G Knowles and R G Moorhouse; Argonne preprint (GUTPA/91/0-2; ANL-HEP-PR-92-01; to appear in Nucl. Phys. B)

12. C D Froggatt, I G Knowles and R G Moorhouse, Phys. Rev. D45 (1992) 2471

13. M Sher, Phys. Rep. 179 (1989) 273;

J Gunion, H E Haber, G L Kane and S Dawson, The Higgs Hunter's Guide, Addison Wesley, 1990

14. CUSB Collaboration, P Franzini et al., Phys. Rev. D35 (1987) 2883

15. S Bertolini, Nucl. Phys. B272 (1986) 77

S Weinberg, Phys. Rev. Lett. 63 (1989) 2333 and Phys. Rev. D42 (1990) 860

S M Barr and A Zee, Phys. Rev. Lett. 65 (1990) 21;

D Chang, W-Y Keung and T C Yuan, Phys. Rev. D43 (1991) 14;

J F Gunion and F Vega, Phys. Lett. B251 (1991) 222;

R C Leigh, S Pabin and R-M Xu, Nucl. Phys. B352 (1991) 45

16. L McLerran, M Shaposhnikov, N Turok and M Voloshin, Phys. Lett. B256 (1991) 451

17. W Bernrewther, T Schröder and T N Pham, Phys. Lett. B279 (1992) 389

C R Schmidt and M E Peskin, Phys. Rev. Lett. 69 (1992) 410

18. P Fayet, Phys. Lett. B64 (1976) 159; B69 (1977) 489;

H P Nilles, Phys. Rep. 110 (1984) 1;

H E Haber and G L Kane, Phys. Rep. 117 (1985) 75

19. P Langacker and M Luo, Phys. Rev. D44, (1991) 817

U Amaldi, W de Boer and H Fürstenau, Phys. Lett. B260, (1991) 447

U Amaldi, W de Boer, P H Frampton, H Furstenau and J T Liu, Phys. Lett. B281 (1992) 374

20. F Anselmo, L Cifarelli, A Peterman and A Zichichi, Nuovo Cimento A104 (1991) 1817

L Clavelli, Phys. Rev. D45 (1992) 3276

J Ellis, S Kelly and D V Nanopoulos, Nucl. Phys. B373 (1992) 55; Phys. Lett. B287 (1992) 85

21. J Bagger, S Dimopoulos and E Masso, Phys. Rev. Lett. 55 (1985) 920

M Olechowski and S Pokorski, Phys. Lett. B257 (1991) 388

22. S Dimopoulos, L J Hall and S Raby, Phys. Rev. Lett. 68 (1992) 1984;

Phys. Rev. D45 (1992) 4192

23. H E Haber and Y Nir, Nucl. Phys. B355 (1990) 363

24. R Barbieri, M Frigeri and F Caravaglios, Phys. Lett. B258 (1991) 167 
25. B Anathanarayan, G Lazarides and Q Shafi, Phys. Rev. D44 (1991) 1663

H Arason et al., Phys. Rev. Lett. 67 (1991) 2933

26. M Carena et al., Nucl. Phys. B369 (1992) 33

27. J E Björkman and D R T Jones, Nucl. Phys. B259 (1985) 533

28. T W Appelquist and J Carrazone, Phys. Rev. D11 (1975) 2856

29. R Tanaka and L Rolandi in talks at the XXVIth ICHEP, Dallas, August 1992

30. G Degrassi, S Franchiotti and A Sirlin, Nucl. Phys. B351 (1992) 49

31. S Bethke, talk at the XXVIth ICHEP, Dallas, August 1992

32. A X el-Khadra, G Hockney, A S Kronfeld and P B Mackenzie, Phys. Rev.Lett. 69 (1992) 729

33. M Kobel, Erlangen University PhD Thesis, DESY-F31-91-03, July 1991

34. BES collaboration: J Z Bai et al., SLAC pre-print: SLAC-PUB-5870, July 1992

35. C D Froggatt and R G Moorhouse, Glasgow University preprint: GUTPA92/07/2

36. J Gasser and H Leutwyler, Phys. Rep. 87 (1982) 77

S Narison, Phys. Lett. B216 (1989) 191

37. Particle Data Group: K Hikasa et al., Phys. Rev. D45 Part II (1992) VI.44

38. M Chanowitz, J Ellis and M K Gaillard, Nucl. Phys. B128 (1977) 506

A J Buras, J Ellis, M K Gaillard and D V Nanopoulos, Nucl. Phys. B135 (1978) 66

39. J Giveon, L J Hall and U Sarid, Phys. Lett. B271 (1991) 138

P H Frampton, J T Liu and M Yamaguchi, Phys. Lett. B277 (1992) 130 


\begin{tabular}{|r|c|c|c|l|l|l|}
\hline Case & $m_{h^{\circ}} \mathrm{GeV}$ & $m_{t} \mathrm{GeV}$ & $m_{b} \mathrm{GeV}$ & $m_{r} \mathrm{GeV}$ & \multicolumn{1}{|c|}{$m_{b} / m_{r}$} & \multicolumn{1}{c|}{$v_{2} \mathrm{GeV}$} \\
\hline $\mathrm{A}$ & $119.2 \pm 9.1$ & $179.3 \pm 14.7$ & $4.20 \pm 0.83$ & $1.78 \pm 0.79$ & $3.19 \pm 1.88$ & 4.25 \\
$1-6 \mathrm{~B}$ & $121.8 \pm 4.3$ & $184.3 \pm 6.8$ & $4.07 \pm 0.33$ & $1.78 \pm 0.33$ & $2.42 \pm 0.74$ & 3.86 \\
$\mathrm{C}$ & $121.7 \pm 4.3$ & $184.3 \pm 6.8$ & $4.30 \pm 1.31$ & 1.78 & $2.42 \pm 0.74$ & $4.03 \pm 0.98$ \\
\hline $\mathrm{A}$ & $123.1 \pm 1.6$ & $186.6 \pm 2.4$ & $4.00 \pm 0.15$ & $1.78 \pm 0.18$ & $2.28 \pm 0.31$ & 3.74 \\
$3-6 \mathrm{~B}$ & $123.2 \pm 0.7$ & $186.8 \pm 1.1$ & $3.97 \pm 0.07$ & $1.78 \pm 0.08$ & $2.24 \pm 0.15$ & 3.69 \\
$\mathrm{C}$ & $123.2 \pm 0.7$ & $186.8 \pm 1.1$ & $3.98 \pm 0.26$ & 1.78 & $2.24 \pm 0.15$ & $3.70 \pm 0.18$ \\
\hline $\mathrm{A}$ & $119.4 \pm 6.9$ & $180.2 \pm 11.1$ & $4.21 \pm 0.54$ & $1.78 \pm 0.54$ & $2.71 \pm 1.13$ & 4.10 \\
$1-3 \mathrm{~B}$ & $120.4 \pm 4.2$ & $182.2 \pm 6.7$ & $4.16 \pm 0.29$ & $1.78 \pm 0.30$ & $2.14 \pm 0.60$ & 3.96 \\
$\mathrm{C}$ & $120.4 \pm 4.2$ & $182.2 \pm 6.7$ & $4.34 \pm 1.07$ & 1.78 & $2.44 \pm 0.60$ & $4.09 \pm 0.78$ \\
\hline
\end{tabular}

Table 1: Fermion and lliggs mass predictions based on $\alpha_{s}=0.125\left(g_{3}=1.253\right)$ and $M_{S}=1$ $\mathrm{TeV}$ using three integer valued starting grids with ranges $(1-6)^{3},(3-6)^{3}$ and $(1-3)^{3}$. The cases $A, B, C$ correspond to the three inethods of tuning $v_{2}$ referred to in the text $(A)$ central value; (B) full statistical average; and (C) point-by-point with statistical average. 


\section{Figure Captions}

Fig. 1 Top, Higgs and bottom mass predictions as a function of the input strong coupling $\alpha_{s}$ $\left(M_{Z}\right)$. The starting grid range was $(1-6)^{3}$ and $M_{s}=1 \mathrm{TeV}$. Method (B) of the text was employed to determine the mean values (solid lines) and standard deviation errors (dotted lines). Note the two vertical scales.

Fig. 2 Top, Higgs and bottom mass predictions as a function of the supersymmetry breaking scale. The starting grid range was $(1-6)^{3}$ with $\alpha_{s}=0.125\left(g_{3}=1.253\right)$. Method (B) of the text was employed to determine the mean values (solid lines) and standard deviation errors (dotted lines). Note the two vertical scales. 


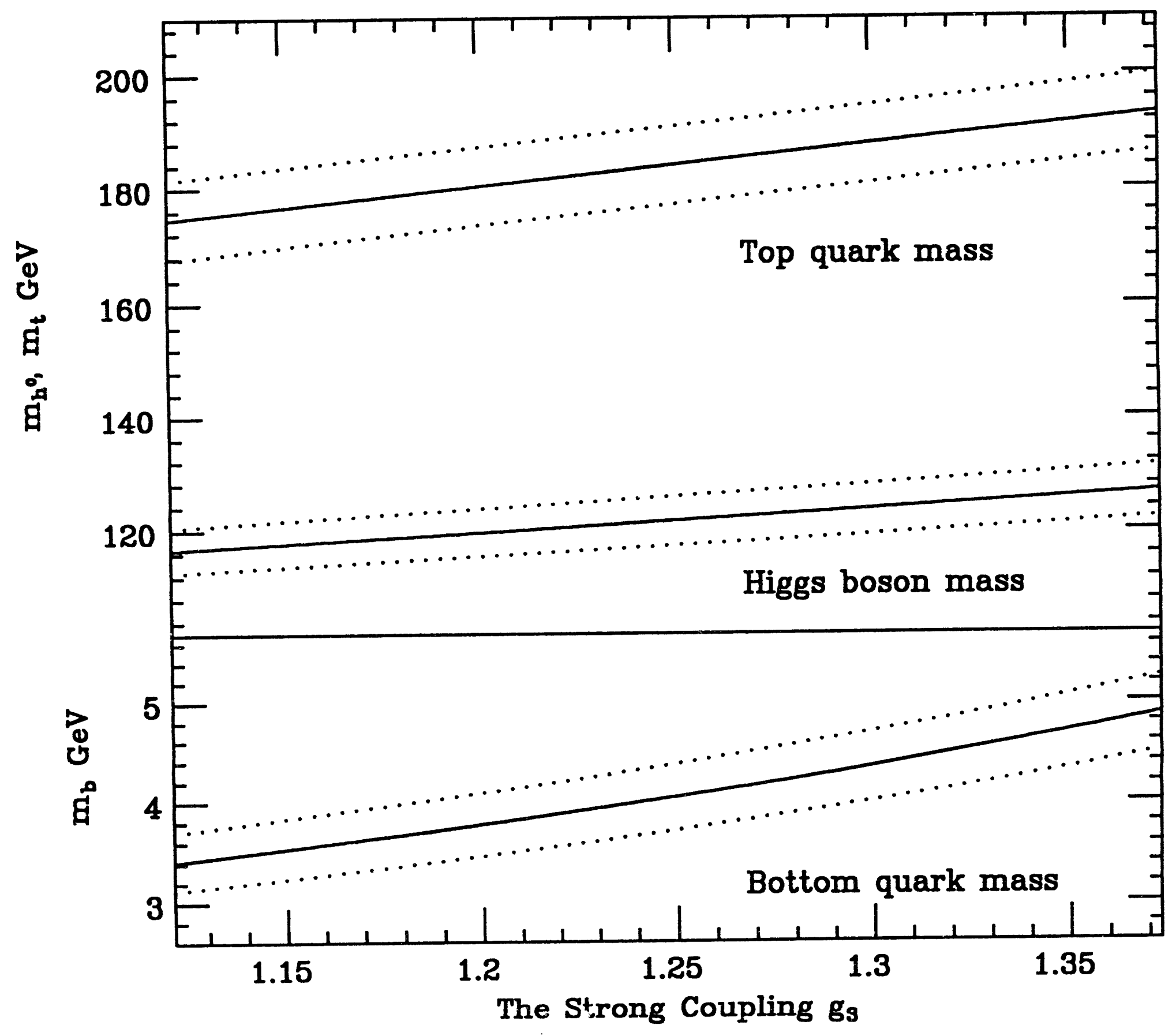

Figure 1 


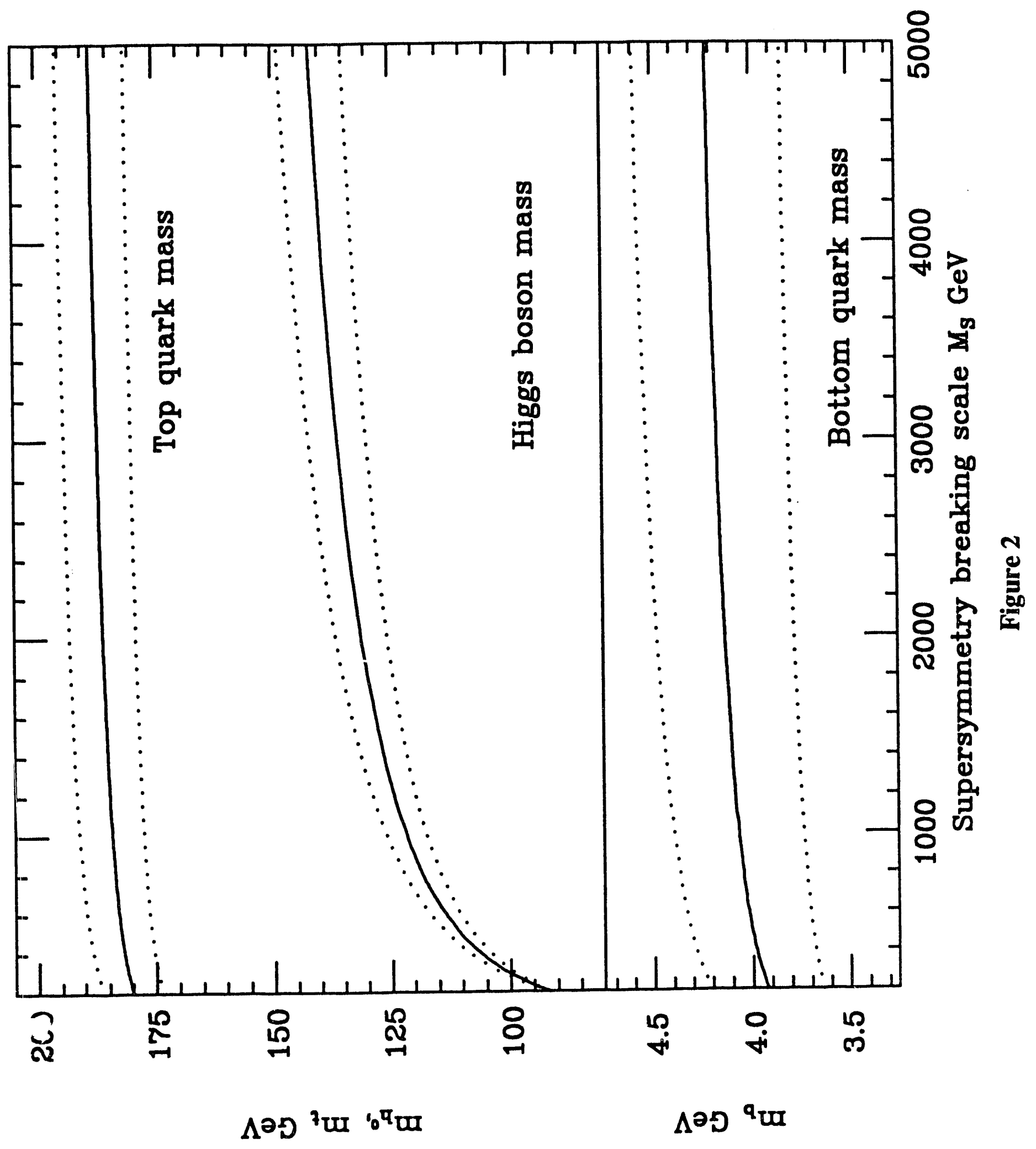


11
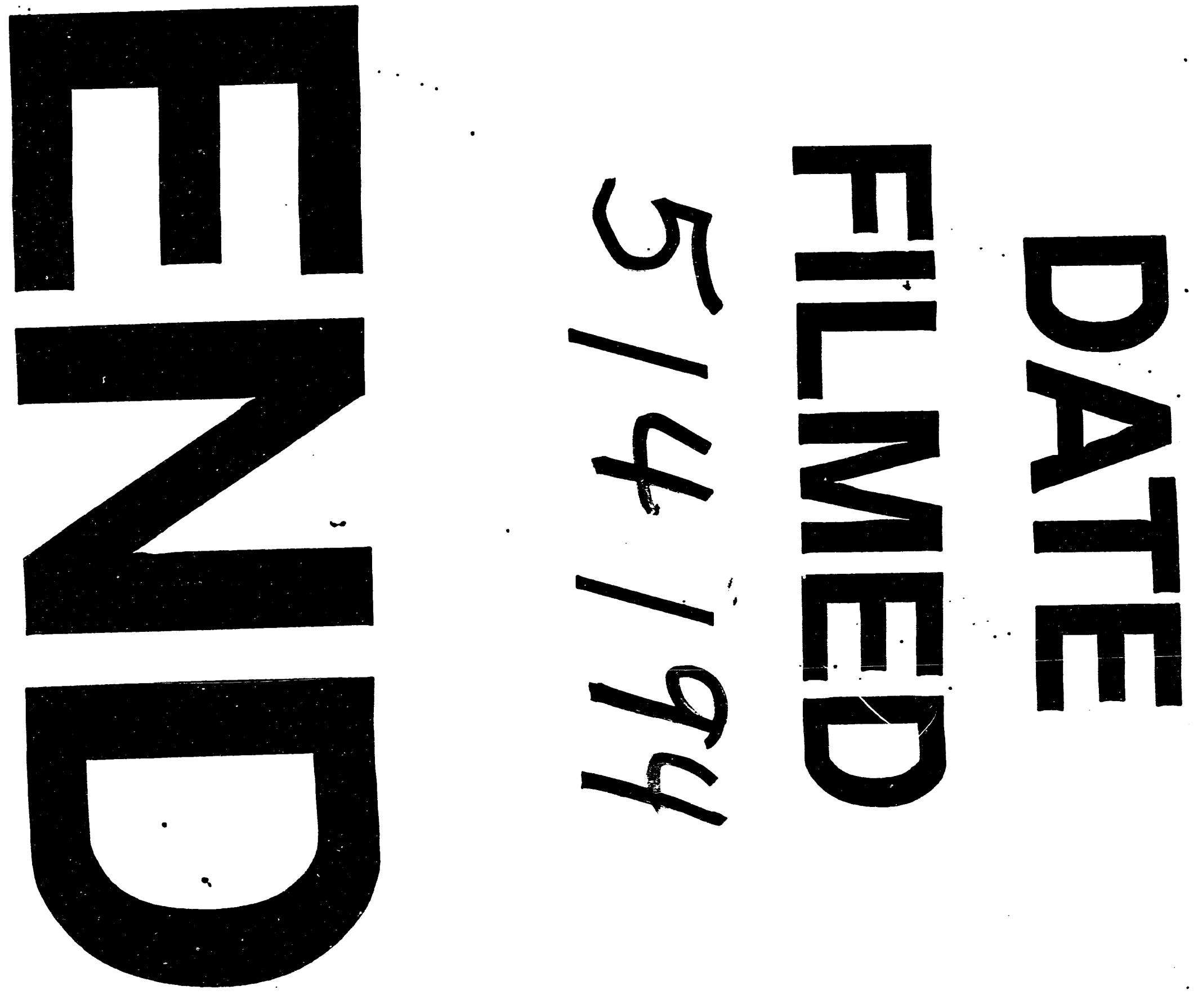


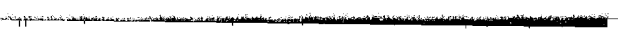

